\title{
Once-weekly prophylactic dosing of recombinant factor IX improves adherence in hemophilia B
}

\author{
This article was published in the following Dove Press journal: \\ Journal of Blood Medicine \\ 30 November 2016 \\ Number of times this article has been viewed
}

\section{Claudia Djambas Khayat \\ Pediatric Department, Hôtel-Dieu de France Hospital, Beirut, Lebanon}

Correspondence: Claudia Djambas Khayat

Pediatric Department, Hôtel-Dieu de France Hospital, Alfred Naccache Boulevard, Beirut, Lebanon

Tel +96I 3704864

Fax +96I I 613273

Email claudiakhayat@yahoo.fr

\begin{abstract}
Regular prophylactic treatment in severe hemophilia should be considered an optimal treatment. There is no general agreement on the optimal prophylaxis regimen, and adherence to prophylaxis is a main challenge due to medical, psychosocial, and cost controversies. Improved approaches in prophylaxis regimen of hemophilia B are needed to make patients' lives easier. There is some evidence to support the efficacy of once-weekly prophylaxis. Longer sampling schedules are required for the determination of pharmacokinetic (PK) properties of factor IX (FIX). The half-life of FIX seems to be longer than previously described and is expected to be 34 hours. The clinical significance of maintaining a $1 \%$ trough level is widely debated in hemophilia B. The overall relationship between factor concentrate levels and incidence of joint bleeding was found to be very weak. Data also indicate that the distribution of FIX into an extravascular FIX compartment may contribute to hemostasis independently of circulating plasma FIX levels. Clinical assessment of the frequency and severity of bleeds remain an important measure of the efficacy of treatment. Role of PK-guided therapy remains to be established. Two prospective randomized studies had evaluated the efficacy and safety of $100 \mathrm{IU} / \mathrm{kg}$ once-weekly prophylaxis with nonacog alfa, and this prophylaxis regimen was found to be associated with lower annual bleeding rate compared with on-demand treatment in adolescents and adults with moderately severeto-severe hemophilia B. Secondary prophylaxis therapy with $100 \mathrm{IU} / \mathrm{kg}$ nonacog alfa once weekly reduced annual bleeding rate by $89.4 \%$ relative to on-demand treatment. Residual FIX may be supportive of effectiveness. Once-weekly prophylaxis was well tolerated in the two studies, with a safety profile similar to that reported during the on-demand treatment period. To individually tailor treatment to clinical response and to minimize costs of factor concentrate, it would be of interest to investigate the efficacy of lower doses of the drug administered once a week.
\end{abstract}

Keywords: hemophilia B, management, prophylaxis, product choice, adherence, administration, dosage

\section{Introduction}

Adherence to treatment is nowadays the main challenge of chronic disease. Hemophilia $\mathrm{B}$ is a chronic hereditary disease characterized by a deficiency in factor IX (FIX) activity. Its prevalence is one in 30,000 live births. It is less common than hemophilia A which is due to factor VIII (FVIII) deficiency. According to the World Federation of Hemophilia Global Survey of 2014, 178,500 persons were identified with hemophilia across 106 countries, of whom only $16.1 \%$ had hemophilia B. ${ }^{1}$

Hemophilia B is an X-linked disease. Missense, frameshift, and nonsense mutations are the most frequently seen, and deletions are rarely described. ${ }^{2}$ 
The severity of the disease is related to clotting factor levels. The disease is classified as severe, moderate, or mild when the clotting factor levels are $<1,1-5$, or $>5$, respectively. ${ }^{3}$ People with mild-to-moderate hemophilia rarely bleed unless after trauma or invasive procedures. Frequency and severity of bleeding is greatest in severe hemophilia B.

Data from the US show that only $36 \%$ of patients with hemophilia B have the severe form of the disease. ${ }^{4}$ Recurrent and spontaneous bleeding into joints and/or soft tissues since early infancy are hallmarks of severity. Gastrointestinal and intracranial bleeding can be life threatening. ${ }^{3}$ Repeated bleeding into joints without adequate treatment can result in crippling chronic joint disease, pain, and reduced quality of life. ${ }^{3,5-8}$

The introduction and availability of factor concentrate has dramatically improved the treatment of hemophilia with a significant decrease in morbidity and mortality, and an increase in quality of life. ${ }^{9}$ Early on-demand treatment of acute bleeding episodes decreases the number of joint deformities compared to untreated or minimally treated patient. ${ }^{10,11}$ Nevertheless, long-term musculoskeletal followup of on-demand treatment in hemophilia showed a progressive deterioration of the joint functions in these patients. As on-demand treatment appears to be clearly suboptimal, prophylaxis - meaning preventive use of clotting factor given at regular intervals - was proposed and adopted since several decades in Sweden. ${ }^{12}$

\section{Prophylaxis}

In 1994, the Medical and Scientific Advisory Council (MASAC) of the US National Hemophilia Foundation issued guidelines stating that prophylaxis with twice- or thrice-weekly FIX infusion, at a dose of 25-40 IU $/ \mathrm{kg}$, should be considered the optimal treatment for hemophilia B. ${ }^{13}$ The European Pediatric Network for Haemophilia Management define primary prophylaxis as regular treatment started after first episode of bleeding and secondary prophylaxis as regular or intermittent regular treatment started after several episodes of bleeding..$^{14,15}$ The World Federation of Hemophila (WFH) guidelines recommend prophylaxis to prevent bleed and joint destruction and preserve normal musculoskeletal function. Moreover, it is advisable to give it to all patients before performing any activities associated with an increased risk of trauma. Prophylaxis should be the state-of-art treatment. ${ }^{3}$ Despite the guidelines, prophylaxis has not been universally adopted. Medical, psychosocial, and cost controversies limit the implementation of prophylaxis..$^{16}$ In the Canadian 2006 survey, only 32\% of patients with severe hemophilia B received prophylaxis, while $69 \%$ of patients with hemophilia A received the treatment. This difference was particularly notable in the $0-2$ years age-group $(17 \%$ in hemophilia $\mathrm{B}$ versus $53 \%$ in hemophilia A) and also present in patients $>18$ years of age ( $20 \%$ versus $55 \%$ ). This difference could be explained by a less severe phenotype, less frequent joint bleeds, later presentation in life, paucity of clinical trials studying the use of prophylaxis, or simply a current treatment tradition. ${ }^{17}$ Moderate hemophilia B is diagnosed much more frequently than the severe (37\% versus $27 \%$ ) form. Positivity to cross-reacting material, which corresponds to measurable level of FIX antigen in plasma, is frequently observed in hemophilia B. This could be explained by the frequency of non-null mutation. Most mutations in hemophilia B are missense mutations and these result in mild-to-moderate form of the disease and account for variable factor levels in plasma. ${ }^{18}$

Studies reporting prophylactic use of FIX in hemophilia B patients are limited in number, and concern mainly small populations within larger clinical trials. ${ }^{19-22}$ Benefits of prophylaxis remain questionable even if intuitively the tendency is in favor. ${ }^{23,24}$ There is currently no evidence to suggest or refute prophylaxis. ${ }^{25}$ Following a 6-month regimen of prophylaxis with recombinant FIX (rIX), patients achieved significantly meaningful improvements in physical health-related quality of life (HRQoL). Those who switched from intermittent prophylaxis to on-demand prophylaxis experienced improvements in physical and mental HRQoL. ${ }^{26}$

\section{Prophylaxis regimen}

There is no general agreement on the optimal prophylaxis regimen, and some schemes differ from those proposed by the Consensus perspectives on prophylactic therapy for hemophilia, held in London, September 20-21, 2002. ${ }^{14}$ Given the paucity of controlled clinical trials regarding prophylaxis in subjects with hemophilia $\mathrm{B}$, choice of treatment approach is limited to clinical judgment, clinical experience, and interpretation of currently available data based on pharmacokinetics (PK), bleeding phenotype, and type of FIX used.

\section{Impact of pharmacokinetics on FIX prophylaxis regimen}

In recent years, greater attention has been paid to the PK of coagulation factor, dosing intervals, and trough levels. PK parameters had an increasing role in management choice even if there was no clear evidence on clinical efficacy. They are routinely measured in clinical practice to guide treatment dosing, particularly for primary prophylaxis, and in connection with surgery. ${ }^{27}$ In 2011, the European Medicines Agency considered appropriate incremental recovery, half-life, area 
under the curve (AUC), and clearance to be the most important surrogate end points for efficacy of FIX products. ${ }^{28}$

PK parameters of FIX are not well characterized or widely investigated. The PK of FIX are more complicated than those of FVIII, and also differ between plasma-derived and recombinant forms resulting in variation between studies. FIX has a longer half-life in the circulation than FVIII. ${ }^{29}$ According to sampling time, the half-life of FIX could be different. After administration of $75 \mathrm{IU} / \mathrm{kg}$ of nonacog alfa to patients aged 12-61 years, the half-life was reported as 22-24 hours when sampled at 72 hours, ${ }^{30}$ and after administration of $50 \mathrm{IU} / \mathrm{g}$, the half-life was reported as 34 hours when sampled at 96 hours. ${ }^{31}$ A sampling schedule at 48 hours gives shorter half-life. In Powell's study, the residual FIX observed 1 week after administration of FIX is supportive of longer half-life. ${ }^{31}$

Strategy to achieve prophylaxis is to maintain the plasma level of factor activity at or above $1 \mathrm{U} / \mathrm{dL}^{3}$ In current bioassays, accuracy can be expected to be rather poor at the conventional target of $1 \mathrm{U} / \mathrm{dL}$, which is the lower limit of the assays. ${ }^{32}$ This issue with assaying was clearly demonstrated by the discrepancies in factor levels in patients with mild-tomoderate and severe hemophilia in the UK National External Quality Assessment Service data. ${ }^{33}$

Furthermore, measuring plasma FIX activity may not fully reflect the hemostatic efficacy of infused FIX. Experimental data demonstrate the potential availability of clinically significant extravascular stores of FIX. They could act as a reservoir of FIX. FIX is a small protein $(55 \mathrm{kDa})$ with access to both intravascular and extravascular compartments. ${ }^{34} \mathrm{~A}$ number of clinical observations in hemophilia B patients suggest the presence of extravascular "pool" of FIX. Bolus infusion of FIX results in rapid initial loss of FIX from the circulation. Repeated bolus infusions result in rise of trough levels. Continuous infusion of FIX results in reduced dose requirement of FIX to maintain a level of $100 \%{ }^{35}$

Furthermore, there are experimental data demonstrating that FIX binds to collagen IV. This may be a source of hemostatically active FIX which is not measurable by plasma assays. ${ }^{34,36}$ Experiments on baboons showed that perfusion of excess bovine FIX increased the circulating FIX proportionally. This suggests the displacement of the host protein from a reservoir. Reanalysis of these data suggests that the extravascular component contains at least threefold more FIX than that present in the circulation. ${ }^{34}$ Two experiments on mouse demonstrated the affinity of FIX to collagen IV and its impact on hemostasis. In a knock-in mouse model expressing a FIX variant with reduced affinity to collagen IV, hemostasis was delayed despite high levels of FIX. Bolus infusion of
FIX variant with enhanced collagen affinity demonstrates a prolonged hemostatic effect in hemophilia B mouse. This hemostatic effect persists days after plasma levels reach less than $1 \%{ }^{37}$ FIX (and FIXa) binds rapidly and reversibly to vascular endothelium and subendothelial extracellular matrix. This is mediated by the interaction of specific residues in the FIX Gla domain with collagen IV, located predominantly in the basement membrane. ${ }^{38,39}$

The clinical significance of maintaining a $1 \%$ trough level is mainly applicable in FVIII deficiency. Such evidence does not exist in FIX deficiency and is widely debated. ${ }^{40}$ In a cohort of 64 patients (51 hemophilia A and 13 hemophilia B) described by Ahnstrom et al, some patients did not bleed with a trough level less than $1 \%$, while others bled with a trough level higher than 3\%. They found that there is no relationship between factor level and incidence of bleed and that the correlation was very weak. Authors suggest that dosing in prophylactic treatment should be individualized. $^{41}$ Due to interindividual variations in PK parameters, targeting a particular trough level may not be appropriate for every individual. ${ }^{41}$ Relationship between FIX trough levels and therapeutic outcomes has not been confirmed in clinical trials. ${ }^{40}$

\section{Impact of hemophilia phenotype on prophylaxis regimen}

Baseline factor levels are not the only determinants of bleeding phenotype in hemophilia. With the same factor level, the severity and frequency of bleeding may be different for people with hemophilia. ${ }^{42}$ In hemophilia B, where limited data exist, there is a need to strike a balance between clinical and PK end points in the evaluation of clinical efficacy. ${ }^{43}$ In hemophilia, bleeding frequency is considered a key indicator of the efficacy of the treatment regimen. The hemophilia severity score (HSS) has been developed as a method to predict bleeding score in hemophilia. ${ }^{44}$ It includes in its assessment the annual joint bleeding rate, annual factor consumption, and WFH orthopedic score. This score was used by Vyas et al to evaluate 178 hemophilia patients without inhibitors in a single US center (hemophilia A [ $n=139]$, hemophilia B $[n=39])$. They found heterogeneity of hemophilia phenotype and widespread variability in the HSS values of patients with the same baseline factor activity. ${ }^{45}$ Data from a single-center cohort study of 171 patients with severe hemophilia A and B in the Netherlands demonstrated the importance of clinical issues in determining the phenotype. They found that age at first joint bleed was an indicator of bleeding pattern. ${ }^{46}$ There was higher annual clotting factor consumption in subjects 
who experienced a joint bleed at an early age comparing to those who experienced later in life. ${ }^{47}$ According to the United Kingdom Haemophilia Centres Doctors' Organisation (UKHCDO) annual report, a large variation in rates of clotting factor concentrate consumption in patients with same diagnosis was also widely observed. ${ }^{48}$

Clinical assessment of the frequency and severity of bleeds remain an important measure of the efficacy of treatment for hemophilia B. The role of PK-guided therapy and its relationship to clinical efficacy remain open issues to be established.

\section{Impact of type of FIX used in prophylaxis regimen}

Historically, effective treatment of hemophilia B started with fresh frozen plasma and prothrombin complex concentrate. In the early 1990 s, highly purified plasma-derived factor concentrate was introduced. ${ }^{49,50}$ Since then, large numbers have become commercially available. ${ }^{51}$ Improvement in sourcing and purification procedure of blood components and the introduction of different steps of viral inactivation increased the safety of plasma-derived concentrates. ${ }^{52}$ Despite these improvements, concerns remain and transfusion-transmitted emerging pathogens like prion and unencapsulated virus should not be overlooked. ${ }^{53-56}$ In 1997 , the first purified rFIX product, nonacog alfa, received US Food and Drug Administration (FDA) approval followed in 2014 by another rFIX product, BAX326. These rFIX products have a lower risk of pathogenic contamination and have low activated FIX activity, which confers low thrombogenic potential in humans. ${ }^{2}$

Traditionally, in vivo recovery (IVR) is the parameter used to characterize the PK properties of coagulation factors. ${ }^{57}$ Following bolus injection, recombinant protein has lower IVR compared to plasma-derived FIX. Terminal half-life is similar for the two products (17-19 hours). ${ }^{40,58,59}$ PK studies that enrolled 308 subjects with hemophilia B in three comparative studies showed that the IVR of rFIX is approximately one-third to one-half that of plasma-derived FIX. ${ }^{58,60,61}$ This disparity in IVR was also noted by Kisker et al in a double-blind crossover study of plasma-derived FIX and rFIX in 15 subjects older than 5 years with severe hemophilia B. ${ }^{62}$ This disparity could be related to baseline FIX levels, body weight, and age. ${ }^{58,63}$ While some individuals have identical recoveries with both types of concentrates, others have pronounced differences. ${ }^{64} \mathrm{~A}$ recent survey in Italy indicated that plasma-derived FIX and rFIX are used in similar doses with similar outcomes. ${ }^{65}$ Despite disparity in recovery, recombinant coagulant factors are nowadays the preferred treatment option for hemophiliacs and, in particular, for pediatric patients mainly in light of the wide margin of safety and efficacy.

\section{Dose and frequency of prophylaxis regimen}

WFH-WHO and NNH propose the twice- or thrice-weekly prophylaxis dose of $50 \mathrm{IU} / \mathrm{kg}$. This regimen was extrapolated from the FVIII prophylaxis experience, early PK data, and the few studies done on FIX. In the original phase 3 nonacog alfa study in previously treated subjects aged $\geq 12$ years, the mean dose was $40.3 \mathrm{IU} / \mathrm{kg}$ administered 2-3 times weekly. ${ }^{22}$ In the phase 3 trial of reformulated nonacog alfa in previously treated subjects, the median dose was $51.7 \mathrm{IU} / \mathrm{kg}$ administered 2-3 times weekly. ${ }^{30}$ Frequency and dose of prophylaxis were determined by the investigator in these two studies. Both the studies demonstrated safety and efficacy in persons with hemophilia B.

However, rigorous regimens of prophylaxis are difficult and adherence remains a problem. ${ }^{66}$ Only $45 \%$ of individuals with severe hemophilia $\mathrm{B}$ are currently receiving prophylaxis in the US. ${ }^{67}$ Cost of clotting factor is certainly a main obstacle for the implementation of such management. It prevents the extensive application of prophylaxis worldwide. It is the largest predictor of overall cost in the care of people with hemophilia. ${ }^{68,69}$ However, the leading reasons for lack of adherence to the prescribed regimen are time consumption, convenient access to peripheral vein, forgetfulness, and the feeling of being healthy with the disappearance of symptoms with time. $^{70,71}$ Many patients find it very difficult to spend 15-20 minutes every morning to mix and infuse intravenous factor. This is certainly more difficult in children where venous access could be difficult and where central venous access devices are associated with the concomitant risk of infection and thrombosis. In an European survey carried out by De Moerloose between October 2005 and September 2006, an interview was conducted with 30 patients in each of six European countries (France, Germany, Italy, Spain, Sweden, and the UK), resulting in a total of 180 patients. Prescribed prophylaxis was not followed in $25 \%$ of patients. The feeling of reduction, fluctuation, or disappearance of symptoms was cited in $38 \%$ of cases, forgetfulness in $36 \%$, lack of time for the treatment in $30 \%$, and convenience in $30 \%$. Forgetfulness was the reason most often cited by patients on prophylaxis $(46 \%) .^{72}$

Therefore, new approaches of management must be proposed to achieve adherence and make patients' lives 
easier. Despite recent promising success in gene therapy for hemophilia B, a cure for hemophilia is not yet available. ${ }^{73,74}$ Novel clotting formulations with longer half-life represent a major advance but at a high financial cost. They are not affordable for most patients with hemophilia. Three studies ${ }^{20,75,76}$ proposed once-weekly prophylaxis with rFIX. It has the potential of increasing convenience to patient, owing to fewer infusions, less preparation and infusion time, and preservation of venous access in those for whom venous access is a challenge. ${ }^{75}$ There is some evidence to support such a regimen. According to Powel's study, residual FIX was observed 1 week after administration of FIX. ${ }^{31}$ Maintaining a $1 \%$ trough level is widely debated in FIX deficiency. ${ }^{40}$ Experimental data demonstrate that FIX has access to both intravascular and extravascular compartments with potential availability of clinically significant extravascular stores of FIX. ${ }^{34}$ Patients with hemophilia B seem to have less severe phenotype and less frequent joint bleed than those with hemophilia A. Vyas et al found heterogeneity of hemophilia phenotype and widespread variability in the HSS values of 178 patients with the same baseline factor activity. ${ }^{45}$

In 1976, Morfini et al published the first randomized trial comparing once-weekly and twice-weekly prophylaxis with on-demand treatment in ten subjects with hemophilia B over 1 year. ${ }^{20}$ A total of $7.5 \mathrm{IU} / \mathrm{kg}$ was administered to the twice-weekly group and $15 \mathrm{IU} / \mathrm{kg}$ to the once-weekly group. The two prophylactic groups had a significant reduction in bleeding episodes when compared to the observed 1-year period preceding the trial $(P<0.005)$. Twice-weekly prophylaxis was superior to once-weekly regimen $(P<0.01)$. Patients having measurable FIX in plasma for a higher number of days bleed less. ${ }^{20}$

In 2014, Valentino et al published a phase 4 multicenter, randomized, open-label, four-period crossover study that evaluated the efficacy and safety of nonacog alfa as a prophylaxis regimen $(50 \mathrm{IU} / \mathrm{kg}$ twice-weekly or $100 \mathrm{IU} / \mathrm{kg}$ once-weekly) compared with on-demand administration. ${ }^{75}$ Inclusion criteria were being male, aged 6-65 years, with severe-to-moderately severe hemophilia (FIX $\leq 2)$, and 12 or more bleeding episodes, including six or more hemarthroses episodes. Fifty patients were enrolled within 12-month participation. The primary end point was the annual bleeding rate $(\mathrm{ABR})$ of two prophylactic regimens compared to on-demand treatment. The ABR was 35.1, 2.6, and 4.6 for the on-demand treatment, $50 \mathrm{IU} / \mathrm{kg}$ twice a week, and $100 \mathrm{IU} / \mathrm{kg}$ once a week, respectively. A significant difference was observed between the two prophylaxis groups and the on-demand group $(P<0.0001)$ with a reduction of bleed of $89.4 \%$ in favor of the prophylaxis group. No difference was observed between the two prophylaxis groups $(P=0.22)$. No patient discontinued the prophylactic treatment because of inefficacy or less-than-expected effect. There was no serious adverse event, or report of thrombogenicity, or occurrence of inhibitors. ${ }^{75}$

The use of high dose of FIX (100 IU/kg per dose) was of concern as it resulted in thrombogenicity. In a review conducted by Rendo et al of five clinical trials in patients with hemophilia $B$, patients who received $\geq 100 \mathrm{IU} / \mathrm{kg}$ per dose of nonacog alfa reported no thrombotic events based on clinical findings and laboratory markers such as thrombinantithrombin (TAT) and D-dimers. ${ }^{77}$ This finding was also confirmed in a study conducted by Kavakli et al. This study evaluated the efficacy and safety of once-weekly prophylaxis with $100 \mathrm{IU} / \mathrm{kg}$ nonacog alfa compared with on-demand treatment in 25 adolescent and adult patients for a period of 52 weeks. ${ }^{76}$ At visits 2 and 4 of the study, assessment of thrombosis was done with monitoring of D-dimers and TAT III complex. Incremental changes in D-dimers and TAT were observed in some patients. None of the patients developed clinical thrombotic event and there was no inhibitor occurrence. Otherwise, mean $\mathrm{ABR}$ was significantly lower in the prophylactic group comparing to the on-demand treatment group. There was 3.6 bleeding events for the prophylactic group and 32.9 events for the on-demand group $(P<0.0001)$. Most interestingly, eight of 17 patients had a FIX superior to $2 \mathrm{U} / \mathrm{dL} 1$ week after dosing. No severe adverse event was observed and once-weekly prophylaxis was well tolerated. ${ }^{77}$

The studies of Valentino ${ }^{75}$ and Kavakli ${ }^{76}$ showed efficacy of once-weekly prophylaxis with no unexpected safety issues, no thrombotic event, no occurrence of inhibitors, and significant reduction in bleeding episodes. However, these two studies had limitations. The population size of the studies was small, although this is quite frequent in hemophilia B studies. Forty-one patients were evaluated in the twice-weekly prophylaxis group of the Valantino study and 25 patients in the Kavakli study. Besides, the study conducted by Valantino was of short duration. Bleed analysis was done during the follow-up period of 16 weeks. This is relatively a too short period to confirm safety and efficacy of a product. The Kavakli study was conducted over a 52-week period and supports safety and efficacy of this regimen in a better way.

Otherwise, the two studies were conducted in previously treated patients and $92 \%$ of the patients included in the Kavakli study had tertiary prophylaxis. The benefit of such regimen in untreated patient or in primary prophylaxis or secondary prophylaxis remains to be demonstrated. Effect of switching from twice-weekly to once-weekly prophylaxis is not yet known. 


\section{Conclusion}

Optimal therapy for patients with hemophilia B is prophylaxis. The current unmet challenges with regard to management of hemophilia B are financial sustainability, inhibitor occurrence, and poor uptake of prophylaxis. Prophylactic regimen must be more convenient to patients and their caregivers, while being effective and safe to improve adherence to treatment. Further research is needed to identify ideal prophylaxis regimens for patients with varying severity of hemophilia B. Once-weekly prophylaxis with rIX may be a viable treatment option for patients with hemophilia B. Prophylaxis regimen at $100 \mathrm{IU} / \mathrm{kg}$ may be a safe and effective alternative to twice-weekly prophylaxis at $50 \mathrm{IU} / \mathrm{kg}$. However, it remains unknown how patients with a less severe bleeding phenotype or with better baseline joint status might respond to once-weekly dosing with rIX. In many regions of the world, FIX doses less than the standard $50 \mathrm{IU} / \mathrm{kg}$ twice-weekly dose are followed. To minimize the cost of treatment and to tailor individual treatment to clinical response, it would be of interest to investigate the effect of once-weekly administration of lower doses of FIX.

\section{Disclosure}

The author reports no conflicts of interest in this work.

\section{References}

1. World Federation of Hemophilia. World Federation of Hemophilia Report on the Annual Global Survey 2014. Montreal, QC: World Federation of Hemophilia; 2014.

2. Franchini M, Frattini F, Crestani S, Sissa C, Bonfanti C. Treatment of hemophilia B:focus on recombinant factor IX. Biologics. 2013;7:33-38.

3. Srivastava A, Brewer AK, Mauser-Bunschoten EP, et al; Treatment Guidelines Working Group on Behalf of the World Federation of Hemophilia. Guidelines for the management of hemophilia. Haemophilia. 2013;19(1):e1-e47.

4. CDC. Universal Data Collection (UDC) System. Atlanta, GA: US Centers for Disease Control and Prevention; 2011.

5. Elander J, Barry T. Analgesic use and pain coping among patients with haemophilia. Haemophilia. 2003;9(2):202-213.

6. Miners AH, Sabin CA, Tolley KH, Jenkinson C, Kind P, Lee CA. Assessing health-related quality-of-life in individuals with haemophilia. Haemophilia. 1999;5(6):378-385.

7. Molho P, Rollan N, Lebrun T, et al. Epidemiological survey of the orthopaedic status of severe haemophilia A and B patients in France. Haemophilia. 2000;6(1):23-32.

8. Aznar JA, Magallón M, Querol F, Gorina E, Tusell JM. The orthopaedic status of severe haemophiliacs in Spain. Haemophilia. 2000;6(3): 170-176.

9. Lusher JM. Prophylaxis in children with hemophilia: is it the optimal treatment? Thromb Haemost. 1997;78 (1):726-729.

10. Ahlberg A. Haemophilia in Sweden VII. Incidence, treatment and prophylaxis of arthropathy and other musculoskeletal manifestations of haemophilia A and B. Acta Orthop Scand Suppl. 1965;77(Suppl):3-132.

11. Hilgartner MW. Hemophilic arthropathy. Adv Pediatr. 1974;21:139-165.

12. Nilsson IM, Hedner U, Ahlberg A. Hemophilia prophylaxis in Sweden. Acta Paediatr Scand. 1976;65(2):129-135.
13. The National Hemophilia Foundation. Medical and Scientific Advisory Council recommendations Concerning Prophylaxis [abstract]. Medical Bulletin 193, Chapter Advisory No197; New York, NY: The National Hemophilia Foundation; 1994.

14. Berntorp E, Astermark J, Bjorkman S, et al. Consensus perspectives in prophylactic therapy for haemophilia: summary statement. Haemophilia. 2003;9(Suppl 1):1-4.

15. Ljiung R, Aranis-Vournas S, Kurnik-Auberger K, et al. Treatment of children with haemophilia in Europe: a survey of 20 centres in 16 countries. Haemophilia. 2000;6(6):619-624.

16. Blanchette VS, Manco-Johnson M, Santagostino E, Ljung R. Optimizing factor prophylaxis for the haemophilia population: where do we stand? Haemophilia. 2004;10(Suppl 4):97-104.

17. Biss TT, Chan AK, Blanchette VS, Iwenofu LN, McLimont M, Carcao MD; Association of Hemophilia Clinic Directors of Canada (AHCDC); Canadian Association of Nurses in Hemophilia Care (CANHC). The use of prophylaxis in 2663 children and adults with haemophilia: results of the 2006 Canadian national haemophilia prophylaxis survey. Hemophilia. 2008;14(5):923-930.

18. Ljung R, Petrini P, Tengborn L, Sjorin E. Haemophilia B mutations in Sweden: apopulation-based study of mutational heterogeneity. $\mathrm{Br}$ J Haematol. 2001;113(1):81-86.

19. Nilsson IM, Berntorp E, Lofqvist T, Pettersson H. Twenty-five years' experience of prophylactic treatment in severe haemophilia A and B. J Intern Med. 1992;232(1):25-32.

20. Morfini M, Mannucci PM, Mariani G, et al. Evaluation of prophylactic replacement therapy in haemophilia B. Scand J Haematol. 1976; 16(1):41-47.

21. Tagliaferri A, Rivolta GF, Rossetti G, Pattacini C, Gandini G, Franchini M. Experience of secondary prophylaxis in 20 adolescent and adult Italian hemophiliacs. Thromb Haemost. 2006;96(4):542-543.

22. Roth DA, Kessler CM, Pasi KJ, Rup B, Courter SG, Tubridy KL; Recombinant Factor IX Study Group. Human recombinant factor IX: safety and efficacy studiesin hemophilia B patients previously treated with plasma-derived factor IX concentrates. Blood. 2001;98(13):3600-3666.

23. Stobart K, Iorio A, Wu JK. Clotting factor concentrates given to prevent bleeding and bleeding-related complications in people with hemophilia A or B. Cochrane Database Syst Rev. 2006;2:CD003429.

24. White GC 2nd. Parachutes and prophylaxis: they both work! J Thromb Haemost. 2006;4(6):1226-1227.

25. Mannucci PM, Franchini M. Is haemophilia B less severe than haemophilia A? Haemophilia. 2013;19(4): 499-502.

26. Windyga J, Lin VW, Epstein JD, et al. Improvement in health-related quality of life with recombinant factor IX prophylaxis in severe or moderately severe haemophilia B patients: results from the BAX326 Pivotal Study. Haemophilia. 2014;20(3):362-368.

27. Berntorp E. Pharmacokinetics of plasma derived and recombinant factor IX: using population pharmacokinetics with sparse sampling data needs further study. Haemophilia. 2013;19(6):805-807.

28. EMA. CHMP guideline on the clinical investigation of recombinant and human plasma-derived factor IX products. EMA/CHMP/ BPWP/144552/2009. London, UK: EMA; 2011.

29. Berntorp E. The pharmacokinetics of clotting factor therapy. Haemophilia. 2003;9(4):353-359.

30. Lambert T, Recht M, Valentino LA, et al. Reformulated BeneFix: efficacy and safety in previously treated patients with moderately severe to severe haemophilia B. Haemophilia. 2007;13(3):233-243.

31. Powell JS, Pasi KJ, Ragni MV, et al. Phase 3 study of recombinant factor IX Fc fusion protein in hemophilia B. $N$ Engl J Med. 2013;369(24):2313-2323.

32. Bjorkman S. Prophylactic dosing of factor VIII and factor IX from a clinical pharmacokinetic perspective. Haemophilia. 2003;9(Suppl 1): 101-108.

33. UK NEQAS. U.K. National External Quality Assessment Scheme for Blood Coagulation: Report on Survey 207. Sheffield: UK NEQAS for Blood Coagulation; 2014. Available from: http:/www.ukneqasbc.org/ Documents/S207\%20report\%20v1.0(1).pdf. Accessed October 11, 2016. 
34. Feng D, Stafford KA, Broze GJ, Stafford DW. Evidence of clinically significant extravascular stores of factor IX. J Thromb Haemost. 2013;11(12):2176-2178.

35. Nazeef M, Sheehan JP. New developments in the management of moderate-to-severe hemophilia B. J Blood Med. 2016;7:27-38.

36. Stern DM, Knitter G, Kisiel W, Nawroth PP. In vivo evidence of intravascular binding sites for coagulation factor IX. Br J Haematol. 1987;66(2):227-232.

37. Gui T, Reheman A, Ni H, et al. Abnormal hemostasis in a knock-in mouse carrying a variant of factor IX with impaired binding to collagen type IV. J Thromb Haemost. 2009;7(11):1843-1851.

38. Gui T, Lin HF, Jin DY, et al. Circulating and binding characteristics of wild-type factor IX and certain Gla domain mutants in vivo. Blood. 2002;100(1):153-158.

39. Cheung WF, van den Born J, Kühn K, Kjellén L, Hudson BG, Stafford DW. Identification of the endothelial cell binding site for factor IX. Proc Natl Acad Sci U S A. 1996;93(20):11068-11073.

40. Bjorkman S. Pharmacokinetics of plasma derived and recombinant factor IX - implications for prophylaxis and on-demand therapy. Haemophilia. 2013;19(6):808-813.

41. Ahnstrom J, Berntorp E, Lindvall K, Bjorkman S. A 6-year follow-up of dosing, coagulation factor levels and bleedings in relation to joint status in the prophylactic treatment of haemophilia. Haemophilia. 2004;10(6): 689-697.

42. Santagostino E, Mancuso ME, Tripodi A, et al. Severe hemophilia with mild bleeding phenotype: molecular characterization and global coagulation profile. J Thromb Haemost. 2010;8(4):737-743.

43. Berntrop E, Dolan G, Hermans C, Laffan M, Santagostino E, Tiede A. Pharmacokinetics, phenotype and product choice in haemophilia B: how to strike a balance? Haemophilia. 2014;20(Supp1 7):1-11.

44. Schulman S, Eelde A, Holmstrom M, Stahlberg G, Odeberg J, Blomback M. Validation of a composite score for clinical severity of Hemophilia. J Thromb Haemost. 2008;6(7):1113-1121.

45. Vyas S, Enockson C, Hernandez L, Valentino LA. Towards personalizing haemophilia care: using the Haemophilia Severity Score to assess 178 patients in a single institution. Haemophilia. 2014;20(1):9-14.

46. Pettersson H, Ahlberg A, Nilsson IM. Aradiologic classification of hemophilicarthropathy. Clin Orthop Relat Res. 1980;149:153-159.

47. Van Dijk K, Fischer K, van der Bom JG, Grobbee DE, van den Berg HM. Variability in clinical phenotype of severe haemophilia:the role of the first joint bleed. Haemophilia. 2005;11(5):438-443.

48. UKHCDO annual report. Available from: http://www.ukhcdo.org/ wp-content/uploads/uploads/216/02/bleeding-disordre-statistics-forApril-2014-to-March-2015-v2-for-website.pdf. Accessed July 182014

49. Kim HC, McMillan CW, White GC, Bergman GE, Horton MW, Said P. Purified factor IX using monoclonal immunoaffinity technique: clinical trials in hemophilia B and comparison to prothrombin complex concentrates. Blood. 1992;79(3):568-575.

50. Furie B, Limentani SA, Rosenfield CG. A practical guide to the evaluation and treatment of hemophilia. Blood. 1994;84(1):3-9.

51. Brooker M. Registry of Clotting Factor Concentrates. 9th ed. Report No. 6. Montreal, QC: World Federation of hemophilia; 2012.

52. Kim IS, Choi YW, Kang Y, Sung HM, Sohn KW, Kim YS. Improvement of virus safety of an antihemophilic factor IX by virus filtration process. J Microbiol Biotechnol. 2008;18(7):1317-1325.

53. Ludlam CA, Turner ML. Managing the risk of transmission of variant Creutzfeldt Jakob disease by blood products. $\mathrm{Br}$ J Haematol. 2006;132(1):13-24.

54. Key NS, Negrier C. Coagulation factor concentrates: past, present, and future. Lancet. 2007;370(9585):439-448.

55. Dietrich B, Schiviz A, Hoellriegl W, et al. Preclinical safety and efficacy of a new recombinant FIX drug product for treatment of hemophilia B. Int J Hematol. 2013;98(5):525-532.

56. Di Minno G, Canaro M, Ironside JW, et al. Pathogen safety of longterm treatments for bleeding disorders: still relevant to current practice. Haematologica. 2013;98(10):1495-1498.
57. Bjorkman S, Folkesson A, Berntorp E. In vivo recovery of factor VIII and factor IX: intra- and interindividual variance in a clinical setting. Haemophilia. 2007;13(1):2-8.

58. Ewenstein BM, Joist JH, Shapiro AD, et al. Pharmacokinetic analysis of plasma-derived and recombinant F IX concentrates in previously treated patients with moderate or severe hemophilia B. Transfusion. 2002;42(2):190-197.

59. Alamelu J, Bevan D, Sorensen B, Rangarajan S. Pharmacokinetic and pharmacodynamic properties of plasma-derived vs. recombinant factor IX in patients with hemophilia B: a prospective crossover study. J Thromb Haemost. 2014;12(12):2044-2048.

60. White GC 2nd, Beebe A, Nielsen B. Recombinant factor IX. Thromb Haemost. 1997;78(1):261-265.

61. Poon MC, Lillicrap D, Hensman C, Card R, Scully MF. Recombinant factor IX recovery and inhibitor safety: a Canadian post-licensure surveillance study. Thromb Haemost. 2002;87(3):431-435.

62. Kisker CT, Eisberg A, Schwartz B; Mononine Study Group. Prophylaxis in factor IX deficiency product and patient variation. Haemophilia. 2003;9(3):279-284.

63. Poon MC. Pharmacokinetics of factors IX, recombinant activated factor VII and factor XIII. Haemophilia. 2006;12(Suppl 4):61-69.

64. Zakarija A. Factor IX replacement in surgery and prophylaxis. Blood Coagul Fibrinolysis. 2004;15(Suppl 2):S5-S7.

65. Rocca A, Pizzinelli S, Oliovecchio E, Santagostino E, Rocino A, Iorio A; Italian ad hoc study group. Replacement therapy with recombinant factor IX. A multicentre evaluation of current dosing practicesin Italy. Blood Transfus. 2011;9(1):60-69.

66. Hacker MR, Geraghty S, Manco-Johnson M. Barriers to compliance with prophylaxis therapy in haemophilia. Haemophilia. 2001;7(4):392-396.

67. Centers for Disease Control and Prevention: Summary Report of UDC Activity NationalTreatment/Clinical Characteristics (Hemophilia) - Report of 31 December, 2011. Available from: https://www2a.cdc. gov/ncbddd/htcweb/UDC_Report/UDC_view1.asp?para1=NATION\& para $2=$ TREATH $\&$ para3 $=\&$ ScreenWidth $=1920 \&$ ScreenHeight $=1080$. Accessed October 19, 2016.

68. Gater A, Thomson TA, Strandberg-Larsen M. Haemophilia B: impact on patients and economic burden of disease. Thromb Haemost. 2011;106(3):398-404.

69. De Moerloose P, Urbancik W, Van Den Berg HM, Richards M. A survey of adherence to haemophilia therapy in sixuropean countries: results and recommendations. Haemophilia. 2008;14(5):931-938.

70. Miners AH, Lee CA. Setting research priorities to improve cost-effectiveness estimations of primary prophylaxis with clotting factor for people with severe haemophilia A. Haemophilia. 2004;10(Suppl 1):58-62.

71. Miners A. Revisiting the cost-effectiveness of primary prophylaxis with clotting factor for the treatment of severe haemophilia A. Haemophilia. 2009;15(4):881-887.

72. Manco-Johnson MJ, Riske B, Kasper CK. Advances in care of children with hemophilia. Semin Thromb Hemost. 2003;29(6):585-594.

73. Nathwani AC, Tuddenham EG, Rangarajan S, et al. Adenovirus-associated virus vector-mediated gene transfer in hemophilia B. $N$ Engl J Med. 2011;365(25):2357-2365.

74. Nathwani AC, Reiss UM, Tuddenham EG, et al. Long-term safety and efficacy of factor IX gene therapy in hemophilia B. $N$ Engl J Med. 2014;371(21):1994-2004.

75. Valentino LA, Rusen L, Elezovic I, Smith LM, Korth-Bradley JM, Rendo P. Multicentre, randomized, open-label study of ondemand treatment with two prophylaxis regimens of recombinant coagulation factor IX in haemophilia B subjects. Haemophilia. 2014;20(3):398-406.

76. Kavakli K, Smith L, Kuliczkowski K, et al. Once-weekly prophylactic treatment vs. on-demand treatment with nonacog alfa in patients with moderately severe to severe haemophilia B. Haemophilia. 2016;22(3):381-388.

77. Rendo P, Shafer FE, Charnigo RJ, Carr M. One-year assessment of coagulation markers and monitoring for thrombotic events in hemophilia $B$ patients treated with nonacog alfa [abstract]. Presented at: World Federation of Hemophilia World Congress; July 8-12; 2012; Paris, France. 
The Journal of Blood Medicine is an international, peer-reviewed, open access, online journal publishing laboratory, experimental and clinical aspects of all aspect pertaining to blood based medicine including but not limited to: Transfusion Medicine; Blood collection, Donor issues, Transmittable diseases, and Blood banking logistics; Immunohematology; Artificial and alternative
Dovepress

blood based therapeutics; Hematology; Biotechnology/nanotechnology of blood related medicine; Legal aspects of blood medicine; Historical perspectives. The manuscript management system is completely online and includes a very quick and fair peer-review system. Visit http://www.dovepress.com/ testimonials.php to read real quotes from published authors. 